\title{
The quantal theory of immunity
}

\author{
Kendall A Smith ${ }^{1}$ \\ ${ }^{1}$ The Division of Immunology, Department of Medicine, Weill Medical College, Cornell University, New York, NY 10021, USA
}

Exactly how the immune system discriminates between all environmental antigens to which it reacts vs. all selfantigens to which it does not, is a principal unanswered question in immunology. As set forth in this review, because of the advances in our understanding of the immune system that have occurred in the last 50 years, for the first time it is possible to formulate a new theory, termed the "Quantal Theory of Immunity", which reduces the problem from the immune system as a whole, to the individual cells comprising the system, and finally to a molecular explanation as to how the system behaves as it does.

Cell Research (2006) 16:11-19. doi:10.1038/sj.cr.7310003; published online 16 January 2006

Keywords: self:nonself recognition, immune system, interleukin-2 (IL2), T cell antigen receptor (TCR), quantal (all-ornone), macromolecular complex

\section{Introduction}

Almost fifty years ago Sir MacFarlane Burnet proposed the Clonal Selection Theory to explain how the immune system functions [1]. Prior to Burnet's theory, Neils Jerne had proposed a natural selection theory in attempt to explain antibody formation [2], and Burnet's contribution was in part an extension of the concept of antigen determination of immunity. However, Burnet improved upon Jerne's idea by giving the immune system a cellular basis, proposing that an individual cell is the source of each unique antibody. In addition, Burnet introduced the idea of clonal expansion through proliferation to explain how the immune system can mobilize a huge number of antigen reactive cells from very few antigen-reactive precursors. He also proposed that lymphocytes were the main cells making up the immune system, thereby correcting Jerne's fallacious hypothesis that phagocytic cells ingest antigen-antibody complexes, and then use the ingested antibodies as templates for the formation of additional antibody molecules.

Now, 50 years later it is amazing that Burnet's Clonal

Correspondence: Kendall A Smith

Tel: 1-212-746-4608; Fax: 1-212-746-8167;

E-mail: kasmith@med.cornell.edu
Selection Theory has been experimentally tested, has held up and now is established as fact, so that it is now accepted as one of the natural Laws of biology. Moreover, in the past 50 years enormous strides have been made in our understanding of the cells and the molecules comprising the immune system. We now know that Burnet was largely correct, and lymphocytes are the main cells comprising the adaptive immune system. As well, we have identified at least 4 main types of lymphocytes, T cells, B cells, natural killer (NK) cells, and regulatory T cells (T-Regs). However in addition, the phagocytic mononuclear cells (i.e. blood monocytes and tissue macrophages) participate in the immune reaction by ingesting antigens and differentiating into "professional" Antigen Presenting Cells (APC), of which two main subsets are recognized, plasmacytoid dendritic cells (pDC), and myeloid DCs (mDC). We also know the molecular structures of both antigens and antigen receptors, as well as how the APCs process and present antigens via molecules encoded by the Major Histocompatibility Complex (MHC) to the antigen recognition receptors on the surface of the T cells. Even so, we still do not have an understanding as to how some antigens are recognized and others are not. Thus, it is now appropriate to reach beyond Burnet's Clonal Selection Law to formulate a new theory as to how the immune system functions to recognize and react to all things foreign, and to recognize, but not react to all molecules that make up self (i.e. self-nonself recognition). 
Any new theory that attempts to explain how the immune system functions to discriminate between self and nonself, must obey Burnet's Law of Clonal Selection and clonal expansion at the level of each individual cell. In addition, any new theory must provide a molecular explanation for the behavior of the individual cells comprising the system as a whole. Most important, the theory must account for the whole immune system to react or not to an antigen, be it nonself or self. This should be possible, now that we know the molecular nature of the receptors for antigen, as well as the molecular nature of antigens.

In biology the definition of quantal is given as "relating to a sensitivity response marked by the presence or absence of a definite reaction (i.e. all-or-none)" [3]. Perhaps one of the first and most well known examples of a quantal response is the contraction of muscle in response to stimulation at the neuromuscular junction. Another example is cell fate determination so well known in embryogenesis. These phenomena are really a variation of Erwin Schrodinger's famous "cat experiment", which he proposed to demonstrate the stochastic nature of radioisotope decay [4]. Thus, quantal decisions, like life or death at the macroscopic level of the whole individual organism, when viewed at the population or group level will be seen as probabilistic, so that for example, if a group of cats is exposed to poison gas, some individuals will succumb before others. However, each individual response is quantal. One of the problems with immunology is that many of our experiments have been performed at the population level with whole organisms, or at the level of cell populations, yet we really need to know what determines the behavior of individual cells.

The Quantal Theory of immunity [5] states that individual cells of the immune system recognize and react to antigens (both nonself and self) by proliferating and differentiating into effector cells in an all-or-none (quantal) fashion. Furthermore, the Quantal Theory states that individual cells make this quantal decision only after "counting" the number of triggered antigen receptors, which determines ultimately the number of interleukin 2 receptors (IL2R) triggered. The number of triggered IL2Rs then ordains the quantal decision to progress through the cell cycle, undergo DNA replication and cytokinesis, which is the basis for clonal expansion. When working backward from an expanded antigen-specific cell population, both the breadth and magnitude of the expanded clones must somehow depend upon the critical molecular determinants of the antigen-dependent recognition and response by individual cells. However, how does this occur?

\section{The quantal nature of IL2-promoted T cell cycle progression}

To take a reductionist approach to the question of how the immune system functions, it is helpful to begin with the end, i.e. after the cells have undergone proliferative clonal expansion, having received the molecular signals that result in activation of the antigen-selected cell clones. The discussion to follow is focused on T cells, but the principles are similar for B cells, NK cells and T-Regs, as well.

Twenty years ago the molecular reagents were in place for the first time to begin to define the variables governing IL2-promoted T cell growth. Thus, with the purification of the IL2 molecule to homogeneity [6], the creation of a radiolabeled IL2 binding assay that permitted the quantification of IL2Rs [7], and the generation of monoclonal T cells [8] as well as monoclonal antibodies reactive with both IL2 [6] and IL2Rs [9], the crucial molecular and cellular parameters could finally be quantified.

Studying T cell populations, whether cloned or not, the symmetrically sigmoid IL2 log-dose response curve [10] is familiar to those skilled in pharmacology or toxicology [11], and is indicative of a quantal biological response on the part of the individual cells comprising the population [11]. It was not until Leonard Herzenberg and his co-workers introduced the flow cytometer to immunology [12] that it was possible to study $\mathrm{T}$ cell proliferation at the level of the single cell [13]. Using propidium iodide to stain the DNA content of cells quantitatively, it was immediately obvious that there is a tremendous heterogeneity among individual cells of a population, even a cloned $\mathrm{T}$ cell population. Thus, when an asynchronously proliferating cell population is exposed to different IL2 concentrations spanning $1 \mathrm{pM}-100 \mathrm{pM}$, some cells can respond to very low IL2 concentrations, while others require higher IL2 concentrations. At the $\mathrm{EC}_{50}$, half of the cells have begun to replicate their DNA, while half have not, when assayed after $24 \mathrm{~h}$ of culture [13].

This heterogeneity of cell growth has been seen with all cell populations, including prokaryotes, and all eukaryotes, including yeasts, protozoa, avian, and all mammalian cells studied over the last 70 years [14]. How to account for this heterogeneity? The key for T cells was found when it was possible to quantify the number of IL2Rs expressed by an individual cell using monoclonal antibodies (MoAbs) reactive with the IL2Ra chain and the flow cytometer. A semi-log plot of the fluorescence intensity of IL2R expression vs. cell number revealed what is now familiar to all immunologists; there is a log-normal (i.e. a normal distribution when cell number is plotted against the $\log _{10}$ receptor density or fluorescence intensity from flow cytometry data) distribution of IL2Rs on individual cells comprising the population that spans at least two orders of magnitude [13]. Thus some cells have very few IL2Rs, while others have 100-fold greater numbers of receptors, with most 


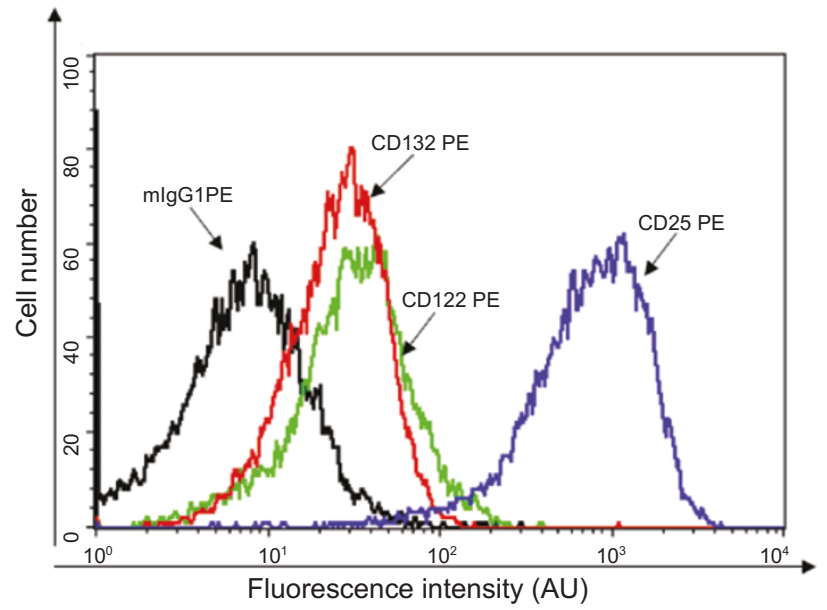

Figure 1 The expression of the three IL2R chains on anti-CD3activated CD4+T cells. Human PBMCs were cultured for $48 \mathrm{~h}$ with anti-CD3 (OKT3; Ortho, $16.7 \mathrm{pM}$ ), then reacted with monoclonal antibodies (BD Biosciences) reactive with CD4, CD25 (IL2R $\alpha$ ), CD122 (IL2R $\beta$ ) and CD132 (IL2R $\gamma_{c}$ ), conjugated with phycoerythrin. $\mathrm{mIgG}-\mathrm{PE}$ is the isotype control antibody. Cell number is plotted on the ordinate in arbitrary units (AU) on a linear scale, while nomoclonal antibody fluorescence intensity is plotted on the abscissa on a $\log$ scale.

cells within the population distributed about the mean in a log-normal fashion between the two extremes. Figure 1 depicts the distribution of the 3 IL2R chains expressed on anti-CD3-activated normal human T cells after $48 \mathrm{~h}$ of culture, when IL2R expression is maximal. It is noteworthy that $\mathrm{CD} 25$ (IL2R $\alpha$ ) expression is $\sim 30$-fold greater than expression of CD122(IL2R $\beta$ ) and CD132(IL2R $\gamma$ ). Once it is realized that cells differ in IL2R expression by orders of magnitude, the obvious question is whether it makes any difference how many IL2Rs an individual cell expresses?

To approach this question, we took advantage of the Fluorescence Activated $\underline{\text { Cell }}$ Sorter (FACS), and separated a cell population into 2 subsets, one with low expression of IL2Rs and one with high IL2R expression, as shown in Figure 2. To assay their response to a receptor-saturating IL2 concentration, we first synchronized the cells into early $G_{1}$ by culture without IL2 for $18 \mathrm{~h}$. It is noteworthy that even $\mathrm{G}_{0 / 1}$ synchronized cells have a log-normal distribution of IL2Rs. After the addition of IL2, cell aliquots were monitored at frequent intervals by short pulses of radiolabeled thymidine. The results were clear-cut; cells with high numbers of IL2Rs traversed the $G_{1}$ phase and entered S-phase more rapidly than cells with fewer IL2Rs [13]. Thus, if a cell has fewer IL2Rs it can still eventually progress through the cell cycle to the S-phase, but it takes longer, as if the cell waits until the "proper" number of
IL2Rs has been triggered.

The other clue as to how the system works came from the variation in the time interval necessary for cells to progress through the cell cycle, which also depended on the density of IL2Rs. Pulse exposure of cells to a receptor-saturating concentration of IL2 for varying time intervals (i.e. 3, 6, 11 , and $24 \mathrm{~h}$ ) revealed that even though IL2/IL2R binding comes to steady-state within $10 \mathrm{~min}$ [15], a minimum of $6 \mathrm{~h}$ was necessary before any cells progressed to S-phase, and those that did so first had the greatest density of IL2Rs [13].

In other experiments we found that the IL2/IL2R complexes formed at the cell surface are internalized and degraded with a $t_{1 / 2}=15 \mathrm{~min}$, while kinetic binding experiments showed that dissociation from the IL2R is three times slower $\left[\mathrm{t}_{1 / 2}=45 \mathrm{~min}\right.$ ] [16]. Thus, once occupied by IL2, the bound IL2R signals until internalized and degraded. Since half of the cells have progressed to S-phase after 11 $\mathrm{h}$ [13], using the $\mathrm{t}_{1 / 2}=15 \mathrm{~min}$ to calculate a rate constant necessary to synthesize new IL2Rs so as to maintain the steady state: $\mathrm{k}=\ln / 15 \mathrm{~min}=0.05 \mathrm{~min}^{-1} \times 11 \mathrm{~h}$ [660 min]
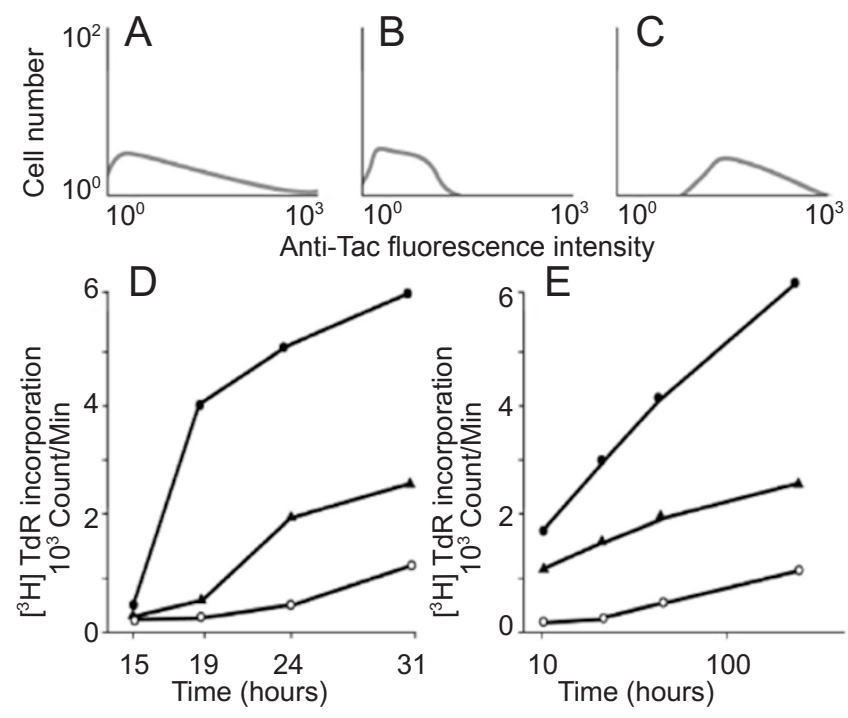

Figure 2 The proliferative response of IL2R + cell populations separated on the basis of IL2R density. Synchronized IL2R+ cells were labeled with anti-Tac (IL2R $\alpha$ ). Viable cells were selected for low or high IL2R $\alpha$ expression via FACS. Flow cytometer analysis of IL2R $\alpha$ expression is shown in the upper panels in linear plots of fluorescence intensity; (A) unseparated cells, (B) low IL2R $\alpha+$ subset, (C) high IL2R $\alpha+$ subset. After sorting, the different cell populations were cultured in the presence of immunoaffinity purified IL2 (0 pM $250 \mathrm{pM})$. (D) Tritiated thymidine $\left[{ }^{3} \mathrm{H}-\mathrm{TdR}\right]$ incorporation in response to a receptor-saturating IL2 concentration $(250 \mathrm{pM})$ of unseparated cells ( $\boldsymbol{\Delta})$, low IL2R $\alpha+\operatorname{subset}(0)$, high IL2R $\alpha+\operatorname{subset}(\bullet)$. (E) $\left[{ }^{3} \mathrm{H}-\right.$ $\mathrm{TdR}$ ] incorporation of the same cell populations as in (D), plotted as a function of IL2 concentration after $31 \mathrm{~h}$ of culture [13]. 
$\times 1,000 \mathrm{Rs} /$ cell $=\sim 33,000 \mathrm{Rs} /$ cell are necessary to trigger DNA replication.

Since the cell population only expresses a mean number of $\sim 1,000$ high affinity IL2Rs/cell [7], it is obvious that new IL2R synthesis must take place over a time interval of several hours. As well, the IL2/IL2R Kd $=\mathrm{EC}_{50}=10^{-11}$ moles $/ \mathrm{L} \times 6.02 \times 10^{23}$ molecules $/$ mole $\times 1 \mathrm{~L} / 1000 \mathrm{~mL}=6$ $\times 10^{9}$ IL2 molecules $/ \mathrm{mL}$ are necessary to half-saturate the IL2Rs. Therefore, it is apparent that the system is constructed so that there is a vast excess of ligand molecules vs. receptor molecules. Moreover, because of receptor limitation, this is an example of a serially triggered ligandreceptor system. That is, the signals resulting from each IL2R occupation on the cell surface somehow accumulate inside the cell sequentially over several hours, which the cell somehow counts, and the cell only progresses to Sphase when the appropriate number of triggered receptors has been accrued.

\section{The structure-activity relationships (SAR) of IL2 and its receptor}

To understand how the IL2Rs trigger a cell in a serial or consecutive fashion, and how the cell senses when the critical number of IL2Rs has been triggered, it is necessary to delineate the SAR of IL2 binding to its receptor. Kinetic IL2 binding studies performed at physiologic temperature $\left(37^{\circ} \mathrm{C}\right)$ using cells that expressed different IL2R chains revealed that the IL2 association rate is very fast, with $\mathrm{k}=$ $10^{7} \mathrm{M}^{-1} \mathrm{~s}^{-1}$ and is attributable to IL2 binding to the $\alpha$-chain (CD25) [15]. By comparison, the rate of dissociation of IL2 from the heterotrimeric IL2R is relatively slow $\left(\mathrm{k}^{\prime}=10^{-4}\right.$ $\mathrm{s}^{-1} ; \mathrm{t}_{1 / 2}=45 \mathrm{~min}$ ), and is primarily attributable to the $\beta$-chain [15]. Since the ratio of $k^{\prime} / k$ specifies the equilibrium dissociation constant $\left(\mathrm{Kd}=10^{-11} \mathrm{M}\right)$, it is readily appreciated that the relatively high affinity of IL2 binding to the trimeric IL2R is conferred by the cooperation between the IL2R $\alpha$ and IL2R $\beta$ chains, each contributing a unique molecular interaction with IL2 [15].

Recent experiments determined the energetics of the interactions between the various IL2R chains and IL2, and revealed that $\alpha / \beta$ heterodimers may form prior to IL2 binding [17] As $\alpha$-chains are expressed at $\sim 30$-fold excess over $\beta$-chains (Figure 1), $\beta$ chains would tend to exist dimerized with $\alpha$ chains [18]. Moreover, the $\beta$-chain cannot interact with the $\gamma$-chain unless IL2 is bound. Thus, it appears that IL2 first binds onto T cells via an $\alpha / \beta$ heterodimeric receptor, forming a trimeric molecular complex, which then favors association with the $\gamma$-chain through a unique entropy-enthalpy compensation mechanism [17]. This sequence of events would certainly make sense, considering that the $\gamma$-chain serves as the critical signaling component of several other structurally distinct cytokines, including IL4, IL7, IL9, IL15, and IL21 [19]. It also fits with IL2 binding data, which have revealed a very low affinity of interaction of IL2 with the $\gamma$-chain itself (i.e. $\sim 700 \mathrm{mM}$ ) [20].

These data have recently been extended by the crystal structure of the fully assembled 3 chains of the IL2 receptor complexed with IL2 [21,22]. The contact area between IL2R $\alpha$ and IL2 reveals a hydrophobic core dominated by 4 IL2R $\alpha$ residues and 5 IL2 residues, and a polar periphery featuring 5 ion pairs, as well as 7 hydrogen bonds. The total contact area between IL2R $\alpha$ and IL2 is large (1590A $\mathrm{A}^{2}$ and $1686 \mathrm{~A}^{2}$ respectively), and the strong electrostatic component of the interaction is consistent with the rapid association rate of IL2-IL2R $\alpha$ binding.

The interface between IL2R $\beta$ and IL2 is the second largest (1151-1159 $\left.\mathrm{A}^{2}\right)$, and this interface features the highest number of hydrogen bonds, which is consistent with its high specificity for IL2 and its relatively slow dissociation rate. By comparison, the interface between IL2R $\gamma$ and IL2 represents the smallest interaction area $\left(971-984 \mathrm{~A}^{2}\right)$, and only 2 hydrogen bonds, thereby providing a structural basis for the low IL2 binding affinity of IL2R $\gamma$.

Perhaps the most striking aspect of the quaternary IL2/IL2R structure is a surprisingly large contact area between the membrane proximal domains of IL2R and IL2R $\gamma\left(1640 \mathrm{~A}^{2}\right.$ and $1611 \mathrm{~A}^{2}$ respectively). There are predominantly polar contacts evenly distributed between IL2R $\beta$ and IL2R $\gamma$, and 14 hydrogen bonds, consistent with a high specificity between the two chains. Accordingly all of this structural information is entirely consistent with the formation of a very stable macromolecular quaternary complex that once formed continues to signal the cell until it is internalized and degraded.

All of these data support the notion that stable macromolecular quaternary IL2/IL2R complexes are formed, which the cell can "count", and it only reacts when a sufficient number have accumulated. However, exactly how the cell performs this mathematical feat remains an enigma.

\section{The intracellular sensors of the IL2/IL2R interac- tion}

More than a decade of research has led to a more complete understanding of the intracellular molecular events triggered by the IL2/IL2R interaction. The IL2R $\beta$ and IL2R $\gamma$ chains have no intrinsic signaling capacity themselves, but both are complexed with members of the Janus tyrosine kinase family (JAK) [23]. Thus, when IL2 binds to IL2R $\alpha \beta$, promoting the IL2R $\beta$ and IL2R $\gamma$ interaction, JAK-1 and JAK-3 are activated, resulting in phosphorylation of themselves as well as their respective receptor chains 
on key tyrosine residues. These chemical changes promote the docking and activation of the Signal Transduction and Activators of Transcription (STAT) 3 and $5 \alpha / \beta$, as well as the adaptor molecule Shc, which activates phosphoinositol 3 kinase (PI3K) [24-26]. These intracellular messengers then cooperate to activate a set of IL2-induced genes, which are responsible for cell cycle progression, differentiation and survival [27-29]. In particular, transcription of the cyclin D2 and D3 genes is induced by IL2, leading to the formation of cyclin $\mathrm{D} / \mathrm{cdk} 4 / 6$ activated complexes, which progressively bind the cyclin/cdk inhibitors $\mathrm{p} 21^{\mathrm{CIP} 1}$ and $\mathrm{p} 27^{\mathrm{kip}}$, reducing their concentrations, and thereby promoting the expression of cyclin $\mathrm{E}$. The $\mathrm{G}_{1}$ cyclin/cdk complexes are responsible for maximally phosphorylating the Retinoblastoma $(\mathrm{Rb})$ proteins, necessary for passage through the $\mathrm{G}_{1}$ restriction point (R-point) [30], ultimately committing the cell irrevocably to DNA replication and mitosis. Accordingly, the decision to pass through the Rpoint in late $\mathrm{G}_{1}$, is by definition, and must be, a quantal decision on the part of each cell.

The timing of the duration of $\mathrm{G}_{1}$ is directly determined by the time it takes to reach the crucial number of triggered IL2Rs. Based upon these considerations, it is obvious that the cell is "hard-wired" from the IL2R to the R-point. It is assumed that the cyclin $\mathrm{D}$ genes are transcribed bi-allelically and continuously in early and mid $\mathrm{G}_{1}$ as a consequence of serial triggering of IL2Rs. In molecular terms, the core decision-making machinery for progression through the $\mathrm{R}$-point to the entrance of S-phase is focused on the phosphorylation of the retinoblastoma proteins $(\mathrm{pRb})$ by the activated cyclinD/cdk4/6 and cyclin E/CDK2 complexes [31-33]. Once phosphorylated completely, $p R b s$ dissociate from members of the family of the E2F transcription factors, thereby allowing them to transcribe the genes required for nucleotide synthesis and DNA replication. However, exactly how this happens in an all-or-none fashion, and whether this occurs instantaneously is unknown. As well, whether a cell must repeat this process with each cell cycle generation is also unknown. That is, do the daughter cells resulting from cytokinesis express IL2Rs, and if so, what is the density of their IL2Rs compared with the mother cell? Alternatively, do the daughter cells need to be triggered again via their antigen receptors to activate IL2R $\alpha$ and $\beta$-chain expression?

\section{The quantal regulation of IL2 gene expression}

Once it is realized that the number of triggered IL2Rs determines quantal $\mathrm{T}$ cell DNA replication and cytokinesis, the next critical molecular question is the nature of the determinants of IL2 production and IL2 gene expression. Accordingly, working backward from the IL2 molecule and the IL2 gene, one is confronted with the T cell antigen receptor (TCR) and the accessory molecules, the costimulatory and coinhibitory receptors, which together promote the expression of the IL2 gene and the IL2R $\alpha$ and $\beta$ genes. Examination of the reports that have focused on how this system functions, it is immediately apparent that the TCR is also triggered serially over several hours, and the signals it generates promote a quantal decision on the part of the cell, just like the IL2R.

The IL2 gene promoter [34] has been studied extensively, and has been found to be regulated via members of 3 distinct families of transcription factors, which include Activating Protein-1 (AP1), Nuclear Factor of Activated $\mathrm{T}$ cells (NF-AT), and Nuclear Factor-kappa B (NF- $\kappa B$ ) [35-38]. Detailed studies by several investigators have shown that the TCR activates NF-AT, AP- 1 and NF- $\kappa B / R e l$ p50/65(RelA). However, activation of the costimulatory molecule CD28 primarily promotes the expression and activation of c-Rel, which is necessary for full and continuous expression of the IL2 gene [39-42]. For example, T cells from c-Rel (-/-) mice are markedly deficient in IL2 gene expression despite having normal expression of NF-AT, AP-1, and NF-kB p50/p65, and supplementation of IL2 exogenously completely circumvents the lack of c-Rel, thereby restoring full proliferative capacity [43]. Therefore, the observation that there are two signals required for full $\mathrm{T}$ cell activation (i.e. via the TCR and the costimulatory molecule CD28) [44] is explained at the level of the IL2 promoter by the necessity of forming a stable macromolecular complex of NF-AT and AP-1, derived from TCR signaling, and c-Rel derived from CD28 signaling.

Individual transcription factors from these 3 families cannot bind stably to their target response elements in the absence of the other distinct factors that bind at neighboring sites $[35,36]$. If a member of any one of these families is prevented from participating, a marked attenuation of IL2 gene transcription occurs. As well, even after the factors have bound, inactivation of any of these three factors pharmacologically extinguishes the binding of all 3 factors, resulting in abortion of transcription. Consequently, for IL2 gene transcription to continue, the TCR and CD28 must deliver tonic signals. Accordingly, it has been proposed [35, $36]$ that there is a nonhierarchical, cooperative enhancement of transcription factor binding at the IL2 gene locus, which results in the formation of a stable macromolecular complex that drives IL2 gene expression in an all-or-none fashion, analogous to the stable macromolecular IL2/IL2R quaternary complex, which serially triggers cyclin D and $\mathrm{E}$ expression, and leads to quantal cell cycle progression.

The importance of signaling via both the TCR and CD28 for the formation a stable macromolecular transcriptional activating complex comes into play when there is absent 
or deficient CD28 signaling; leaving only signals from the TCR (i.e. signal 1 without signal 2). In this case, NF-AT and AP-1 activation predominate, abortive expression of the IL2 gene occurs, and the cells are rendered anergic via the activation of a negative regulatory gene program, as well as the degradation of TCR signaling components, such as PLC $\gamma$ and PKC $\theta$ [45-49].

Supporting the quantal regulation of IL2 gene expression are studies on the allelic expression of the IL2 gene. Under optimal TCR stimulating conditions, when antigen is in excess, and APCs are not limiting, there is biallelic expression of the IL2 genes [50, 51]. However, if TCR stimulatory conditions are suboptimal, then expression is monoallelic, and as the conditions are reduced further, fewer and fewer cells will express the IL2 gene [51, 52]. Accordingly, when examined at the single cell level, one is confronted with the quantal nature of IL2 gene expression, and the realization that whether an individual cell expresses its IL2 genes is a function of the "strength" of the TCR signal [53].

A series of experiments have now revealed that like IL2R signaling [13], the strength of the TCR signal is determined by the antigen concentration, the density of the TCR expressed by an individual cell, and the duration of the antigen/TCR interaction [54-56]. The Supramolecular Activation Cluster (SMAC) [57], or the Immunological Synapse [58] is actually a very complicated and very large stable macromolecular complex that forms between a $T$ cell and an APC [59]. On the T cell, the synapse is comprised of a central cluster of TCRs together with costimulatory and coinhibitory molecules, surrounded by a more peripheral ring of adhesion molecules. In this regard, it is important to note that most of the TCRs and costimulatory molecules expressed by an individual cell participate in the formation of the "central' synapse, so that there would be a mean of $\sim 100,000$ TCRs focused in this one area. However, because of the log-normal distribution of TCRs among cells of a given population, some cells will express only $\sim 10,000$ TCRs while others could express as many as $1,000,000$ TCRs. The APC synapse area is comprised of a central cluster of antigenic peptide bound to MHC-encoded molecules (pMHC), together with the ligands capable of interacting with the costimulatory and coinhibitory molecules expressed by the T cell (i.e. B7.1 \& B7.2) [60]. Surrounding this central cluster are the ligand molecules capable of binding with the adhesion molecules expressed on the T cells. Therefore, it is also important to quantify the total number of MHC molecules expressed by an individual APC, and then also to determine how many MHC molecules have bound an antigenic (non-self) peptide vs. how many have bound endogenous (self) peptides.

Like the density of IL2Rs expressed by T cell clones, the TCR density on individual cells of a clone also varies by 2 orders of magnitude, and there is always a log-normal distribution of TCRs on cells comprising a population, even a cloned, synchronized $\mathrm{T}$ cell population. Thus, there is an individual cell variability of TCR density. Detailed studies examining the cytokine response of murine $\mathrm{T}$ cell clones to graded antigenic peptide concentrations have revealed a hierarchical organization of TCR signal-dependent response thresholds for elicitation of IL2 gene expression by individual cells [51]. As the peptide concentration is increased, the number of cells producing IL2 increases, while the amount of IL2 produced/cell remains constant. Thus, the decision to produce IL2 is a quantal decision on the part of each cell, even in a cloned cell population, which is comprised of genetically homogeneous progeny of a single cell.

How can one account for this heterogeneity of response? Experiments monitoring the production of IFN $\gamma$ by individual cells of a cloned human $\mathrm{T}$ cell population in response to graded concentrations of influenza virus peptide antigen have revealed results that are remarkably similar to the proliferative responses of individual cells of a T cell clone to graded concentrations of IL2 [61]. The peptide concentrations that stimulate $5 \%$ of the cells within the population vs. $95 \%$ of the cells span two orders of magnitude, and the response on the part of the cells comprising the population is quantal; i.e. at low antigen concentrations fewer cells produce IFN $\gamma$, and as the antigen concentration is increased, an increasing number of cells are capable of producing IFN $\gamma$.

The logical explanation for the heterogeneity of response at varying peptide antigen concentrations is that only the cells with the highest density of TCRs are capable of responding to the lowest concentration of peptide. This idea has been tested in vivo by the construction of TCR transgenic mice with regulatable expression of their TCRs [62]. The $\mathrm{pMHC} / \mathrm{TCR}$ system is organized to be very sensitive to low antigen concentrations by the presence of a high density of TCRs. Thus, at a mean TCR density of $\sim 20,000$ TCRs/cell, very low antigenic peptide doses administered intravenously maximally activate IL 2 production and proliferation. Moreover, not until the TCR density is reduced to $<1,000 \mathrm{TCRs} / \mathrm{cell}$ are higher peptide antigen concentrations necessary to drive the response. Accordingly, because there is such a large excess of TCRs, a cell would have to loose expression of many TCRs before it would become unresponsive to even low amounts of antigenic pMHC.

Other studies have made it possible to "count" the exact number of pMHC ligands that a $\mathrm{T}$ cell encounters on an $\mathrm{APC}$, and then monitor the consequences of the TCR/pMHC interaction by quantifying increases in intracellular calcium $[60,63]$. It has been found that as little as $1 \mathrm{pMHC}$ ligand 
presented per APC can trigger a T cell, and only $10 \mathrm{pMHC}$ ligands are sufficient to provide for the formation of a stable immunological synapse for several hours. However, below this critical number of pMHC ligands/APC, only transient calcium increases occur, and a stable synapse does not form. Even so, given the fact that most APCs express $\sim 100,000$ $\mathrm{MHC}$ molecules/cell, the vast excess of MHC molecules must be occupied by endogenous (self) peptides. In this regard, recent studies have indicated that endogenous pMHC molecules and agonist pMHC molecules act cooperatively to amplify TCR signaling $[64,65]$.

In other experiments, peptide specific $\mathrm{T}$ cell clones have been allowed to form a stable synapse with the appropriate pMHC ligands, followed by interruption of the synapse using monoclonal antibodies reactive with the pMHC complex at various time intervals [60]. This approach, coupled with measures of IL2 production as well as proliferation assays by fluorescent dye dilution, have shown that greater than $10 \mathrm{~h}$ of stable synapse formation between the $\mathrm{T}$ cell and APC are required for an optimal response.

Accordingly, just like the IL2R [13], serial pMHC triggering of the TCR leads to a quantal cellular response, but in contrast to the IL2/IL2R system, the pMHC/TCR system is set up in favor of the (pMHC), which can be very low and still trigger a response. Another way of stating this is to realize that there is a great excess of TCRs, $\sim 100,000$ molecules/cell, that can be triggered by very few agonist pMHC molecules, $<10$ molecules/cell. It may very well be that the excess ligand-to-receptor ratio will be found to be characteristic of soluble mediators, while an excess receptor-to-ligand ratio will be characteristic for cell-associated ligand-receptor interactions. Far fewer ligands may be necessary in membrane-tethered signaling because the ligands are membrane-bound and therefore efficiently localized; however, the restriction of diffusion to 2 dimensions may make diffusion faster, necessitating the presence of more receptors to maintain an efficient signaling response.

\section{Summary and conclusions}

All of these data are consistent with the Quantal Theory of Immunity. Like the IL2/IL2R-determined quantal decision to undergo cell cycle progression, there appear to be quantal decisions operative at the level of the immunological synapse that lead to distinct cell fates, which in turn are ultimately determined by the extent and duration of IL 2 and IL2R gene expression. For example, if the agonist pMHC ligand concentration is low, only T cells with a high affinity and density of TCRs will form a stable synapse that can result in sustained triggering of IL2 gene expression, and thereby cell cycle progression, followed by T cell differentiation and survival. Obviously, the initial number of T cell

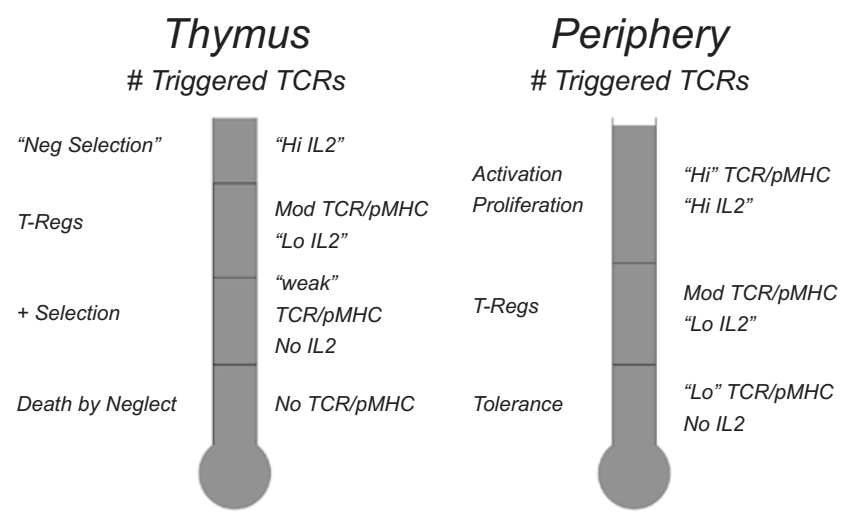

Figure 3 The number of triggered TCRs and IL2Rs determine quantal $\mathrm{T}$ cell fates in both the thymus and the periphery. On each plot, the number of triggered TCRs and IL2Rs increase from bottom to top. The different quantal fates are dictated by a definite number of triggered Rs, as depicted [5].

precursors that are triggered will be reflected ultimately by the number and size of the expanded clones of cells, and this will determine whether there is a detectable systemic immune response. Accordingly, when the pMHC antigen concentration increases, $\mathrm{T}$ cells with lower TCR affinities and/or densities will be brought into play, resulting in a greater number of precursors activated, thereby resulting in a greater systemic immune response. By comparison, should the pMHC concentration be too low, then cells with low TCR affinities and/or densities may have abortive expression of the IL2 gene, which would be predicted to favor differentiation to T-Reg cells [66-69]. Cells with still lower TCR densities when confronted with low pMHC concentrations may become anergic because of the lack of activation of IL2 gene expression [48].

All of these considerations indicate that the fates of cells within the immune system, and thus the fate of the entire system, are determined by the quantity of antigen, and not the quality of antigen. In other words, there is no difference between autoantigens and foreign antigens except their quantity and distribution. This concept is supported by the fact that both autoantigens and foreign antigens are comprised of peptides bound to MHC molecules. It follows that since both autoantigens and foreign antigens are identical in structure, the only possible way that the immune system can discriminate between the two is quantitative. The same principles responsible for self-nonself discrimination in the periphery are also at play within the thymus, as shown in Figure 3: thus, the number of triggered TCRs will determine death by neglect, positive selection, T-Reg differentiation, and negative selection. For a more extensive treatment of the Quantal Theory of Immunity, especially as it relates 
to the maturation of $\mathrm{T}$ cells within the thymus, the reader is referred to [5]: At very low numbers of triggered TCRs in the thymus, cells will die from lack of signal. As the number of triggered TCRs increases, due to an increase in pMHC concentration, TCR affinity or density, cells will be positively selected and exported to the periphery. A further increase in triggered TCRs will promote enough IL2 production to initiate the maturation to T-Regs, which also are exported to the periphery. Finally, at maximal number of triggered TCRs, IL2 production will be maximal, but instead of proliferative expansion as would occur in the periphery, apoptosis results in negative selection. Accordingly, the only cells exported to the periphery are those positively selected to have a low affinity for self-pMHC.

For the future, we must come to a molecular understanding of how the cells "count", and how stable molecular complexes are first formed and then dissociated on cue. For these questions we may well need to involve the principles of biological physics and nonequilibrium reactions. However, at this time it may well be that the same principles that govern the fundamental quantal fates of cells within the immune system, and which then dictate how the entire immune system is able to discriminate self from nonself, are also governing the quantal fates of cells in other biological systems, for example such fundamental cellular fate determinations as those forming the embryo.

\section{Acknowledegment}

The author is grateful for financial support from the Belfer Foundation, and the NIH, NIAID.

\section{References}

1 Burnet FM. A modification of Jerne's theory of antibody production using the concept of clonal selection. Aust J Sci 1957; 20:67.

2 Jerne NK. The natural selection theory of antibody formation. Proc Natl Acad Sci U S A 1955; 41:849.

3 Quantal. http://www.m-w.com/dictionary/quantal

4 Schrodinger E. Cat experiment. 1935; http://www.mtnmath. $\mathrm{com} / \mathrm{faq} / \mathrm{meas}-\mathrm{qm}-3 . \mathrm{html}$

5 Smith KA. The quantal theory of how the immune system discriminates between "self and non-self". Med Immunol 2004; 3:3.

6 Smith KA, Favata MF, Oroszlan S. Production and characterization of monoclonal antibodies to human interleukin 2: strategy and tactics. J Immunol 1983; 131:1808.

7 Robb RJ, Munck A, Smith KA. T cell growth factor receptors. Quantitation, specificity, and biological relevance. J Exp Med 1981; 154:1455.

8 Baker PE, Gillis S, Smith KA. Monoclonal cytolytic T-cell lines. J Exp Med 1979; 149:273.

9 Leonard WJ, Depper JM, Uchiyama T, et al. A monoclonal antibody that appears to recognize the receptor for human T-cell growth factor; partial characterization of the receptor. Nature 1982; 300:267.

10 Gillis S, Ferm MM, Ou W, Smith KA. T cell growth factor: parameters of production and a quantitative microassay for activity. J Immunol 1978; 120:2027.

11 Kane A. Dose-response. http://aquaticpath.umd.edu/appliedtox/ dose-response.pdf

12 Parks DR, Bryan VM, Oi VT, Herzenberg LA. Antigen-Specific Identification and Cloning of Hybridomas with a FluorescenceActivated Cell Sorter. Proc Nat Acad Sci U S A 1979; 76:1962.

13 Cantrell DA, Smith KA. The interleukin-2 T-cell system: a new cell growth model. Science 1984; 224:1312.

14 Pardee A, Shilo B, Koch A. Variability of the cell cycle. In: Hormones and Cell Culture. Sato G, Ross R, eds. Cold Spring Harbor Laboratory, Cold Spring Harbor: NY 1979; 373.

15 Wang HM, Smith KA. The interleukin 2 receptor. Functional consequences of its bimolecular structure. J Exp Med 1987; 166:1055.

16 Smith KA. The interleukin 2 receptor. Annu Rev Cell Biol 1989; 5:397.

17 Rickert M, Boulanger M, Goriatcheva N, Garcia K. Compensatory energetic mechanisms mediating the assembly of signaling complexes between interleukin 2 and its alpha, beta and gamma receptors. J Mol Biol 2004; 339:1115.

18 Smith KA, Cantrell DA. Interleukin 2 regulates its own receptors. Proc Natl Acad Sci U S A 1985; 82:864.

19 Leonard W, Shores E, Love P. Role of the common cytokine receptor gamma chain in cytokine signaling and lymphoid development. Immunol Rev 1995; 148:97.

20 Liparoto SF, Myszka DG, Wu Z, et al. Analysis of the role of the interleukin-2 receptor chain in ligand binding. Biochemistry 2002; 41:2543.

21 Wang X, Rickert M, Garcia KC. Structure of the quaternary complex of interleukin-2 with its alpha, beta, and gammac receptors. Science 2005; 310:1159.

22 Stauber D, Debler E, Horton P, Smith K, Wilson I. Crystal structure of the interleukin-2 signaling complex: paradigm for a heterotrimeric cytokine receptor. Proc Natl Acad Sci U S A 2005 In press.

23 Ihle JN. Cytokine receptor signalling. Nature 1995; 377:591.

24 Martino A, Holmes JH 4th, Lord JD, Moon JJ, Nelson BH. Stat5 and $\mathrm{Sp} 1$ regulate transcription of the cyclin $\mathrm{D} 2$ gene in response to IL-2. J Immunol 2001; 166:1723.

25 Moon JJ, Nelson BH. Phosphatidylinositol 3-kinase potentiates, but does not trigger, $\mathrm{T}$ cell proliferation mediated by the IL-2 receptor. J Immunol 2001; 167:2714.

26 Moon J, Rubio E, Martino A, Krumm A, Nelson B. A permissive role for phophatidylinositol 3-kinase in the STAT5-mediated expression of cyclin D2 by the interleukin-2 receptor. J Biol Chem 2004; 279:5520.

27 Stern JB, Smith KA. Interleukin-2 induction of T-cell G1 progression and c-myb expression. Science 1986; 233:203.

28 Beadling C, Johnson KW, Smith KA. Isolation of interleukin 2induced immediate-early genes. Proc Natl Acad Sci U S A 1993; 90:2719.

29 Beadling C, Smith KA. DNA array analysis of interleukin-2regulated immediate/early genes. Med Immunol 2002; 1:2

30 Pardee A. A restriction point for control of normal animal cell proliferation. Proc Natl Acad Sci U S A 1974; 71:1286.

31 Sherr C. D-type cyclins. Trends in Biochem Sci 1995; 20:187. 
32 Sherr C, Roberts J. CDK inhibitors: positive and negative regulators of G1 progression. Genes \& Development 1999; 13:1501.

33 Sherr C. The pezcoller lecture: cancer cell cycles revisited. Cancer Res 2000; 60:3689.

34 Holbrook NJ, Smith KA, Fornace AJ Jr, et al. T-cell growth factor: complete nucleotide sequence and organization of the gene in normal and malignant cells. Proc Natl Acad Sci U S A 1984; 81:1634.

35 Garrity PA, Chen D, Rothenberg EV, Wold BJ. Interleukin 2 transcription is regulated in vivo at the level of coordinated binding of both constitutive and regulated factors. Molecular and Cellular Biology 1994; 14:2159.

36 Rothenberg EV, Ward SB. A dynamic assembly of diverse transcription factors integrates activation and cell-type information for interleukin 2 gene regulation. Proc Natl Academ Sci U S A 1996; 93:9358.

37 Shaw JP, Utz PJ, Durand DB, et al. Identification of a putative regulator of early T cell activation genes. Science 1988; 241:202.

38 Northrop JP, Ho SN, Chen L, et al. NF-AT components define a family of transcription factors targeted in T cell activation. Nature 1994; 369:497.

39 Fraser JD, Irving BA, Crabtree GR, Weiss A. Regulation of interleukin-2 gene enhancer activity by the $\mathrm{T}$ cell accessory molecule CD28. Science 1991; 251:313.

40 Thompson CB, Lindsten T, Ledbetter JA, et al. CD28 activation pathway regulates the production of multiple T-cell- derived lymphokines/cytokines. Proc Natl Acad Sci U S A 1989; 86:1333.

41 Verweij CL, Geerts M, Aarden LA. Activation of interleukin 2 gene transcription via the T cell surface molecule CD28 is mediated through an NF-kB-like response element. J Biol Chem 1991; 266:14179.

42 Ghosh P, Tan TH, Rice NR, Sica A, Young HA. The interleukin 2 CD28-responsive complex contains at least three members of the NF-kB family: c-Rel, p50, and p65. Proceedings of the National Academy of Sciences USA 1993; 90:1696.

43 Liou H, Jin Z, Tumang J, et al. c-Rel is crucial for lymphocyte proliferation but dispensable for $\mathrm{T}$ cell effector function. Int Immunol 1999; 11:361.

44 Jenkins M, Schwartz R. Antgen presentation by chemically modified splenocytes induces antigen-specific T cell unresponsiveness in vitro and in vivo. J Exp Med 1987; 165:302.

45 Macian F, Garcia-Rodriguez C, Rao A. Gene expression elicited by NFAT in the presence or absence of cooperative recruitment of Fos and Jun. Embo J 2000; 19:4783.

46 Macian F, Lopez-Rodriguez C, Rao A. Partners in transcription: NFAT and AP-1. Oncogene 2001; 20:2476.

47 Macian F, Garcia-Cozar F, Im SH, et al. Transcriptional mechanisms underlying lymphocyte tolerance. Cell 2002; 109:719.

48 Macian F, Im SH, Garcia-Cozar FJ, Rao A. T-cell anergy. Curr Opin Immunol 2004; 16:209.

49 Heissmeyer V, Macian F, Im SH, et al. Calcineurin imposes T cell unresponsiveness through targeted proteolysis of signaling proteins. Nat Immunol 2004; 5:255.

50 Chiodetti L, Barber D, Schwartz R. Biallelic expression of the IL-2 locus under optimal stimulation conditions. Eur J Immunol 2000; 30:2157.
51 Itoh Y, Germain RN. Single cell analysis reveals regulated hierarchical $\mathrm{T}$ cell antigen receptor signaling thresholds and intraclonal heterogeneity for individual cytokine responses of CD4+ T cells. J Exp Med 1997; 186:757.

52 Hemmer B, Stefanova I, Vergelli M, Germain RN, Martin R. Relationships among TCR ligand potency, thresholds for effector function elicitation, and the quality of early signaling events in human T cells. J Immunol 1998; 160:5807.

53 Gett A, Sallusto F, Lanzavecchia A, Geginat J. T cell fitness determined by signal strength. Nat Immunol 2003; 4:355.

54 Viola A, Lanzavecchia A. T cell activation determined by $\mathrm{T}$ cell receptor number and tunable thresholds. Science1996; 273:104.

55 Iezzi G, Karjalainen K, Lanzavecchia A. The duration of antigenic stimulation determines the fate of naive and effector $\mathrm{T}$ cells. Immunity $1998 ; \mathbf{8}: 89$

56 Valitutti S, Muller S, Dessing M, Lanzavecchia A. Different responses are elicited in cytotoxic T lymphocytes by different levels of T cell receptor occupancy. J Exp Med 1996; 183:1917.

57 Monks C, Freiberg B, Kupfer H, Sciaky N, Kupfer A. Three dimensional segregation of supramolecular activation clusters in T cells. Nature 1998; 395:82.

58 Grakoui A, Bromley SK, Sumen C, et al. The immunological synapse: a molecular machine controlling $\mathrm{T}$ cell activation. Science 1999; 285:221.

59 Huppa J, Gleimer M, Sumen C, Davis M. Continuous T cell receptor signaling required for synapse maintenance and full effector potential. Nat Immunol 2003; 4:749.

60 Huppa J, Davis M. T-cell antigen recognition and the immunological synapse. Nat Rev Immunol 2003; 3:973.

61 Hesse MD, Karulin AY, Boehm BO, Lehmann PV, Tary-Lehmann M. A T cell clone's avidity is a function of its activation state. $\mathrm{J}$ Immunol 2001; 167:1353.

62 Labrecque N, Whitfield LS, Obst R, et al. How much TCR does a $\mathrm{T}$ cell need? Immunity 2001; 15:71.

63 Krummel M, Davis M. Dynamics of the immunological synapse: finding, establishing and solidifying a connection. Curr Opin Immunol 2002; 14:66.

64 Li Q, Dinner A, Qi S, et al. CD4 enhances T cell sensitivity to antigen by coordinating Lck accumulation at the immunological synapse. Nat Immunol 2004; 5:791.

65 Krogsgaard M, Li QJ, Sumen C, et al. Agonist/endogenous peptide-MHC heterodimers drive $\mathrm{T}$ cell activation and sensitivity. Nature 2005; 434:238.

66 Thornton AM, Shevach EM. CD4+CD25+ immunoregulatory T cells suppress polyclonal $\mathrm{T}$ cell activation in vitro by inhibiting interleukin 2 production. J Exp Med 1998; 188:287.

67 Jordan MS, Boesteanu A, Reed AJ, et al. Thymic selection of CD4+CD25+ regulatory $T$ cells induced by an agonist self-peptide. $2001 ; \mathbf{2}: 301$.

68 Thornton AM, Donovan EE, Piccirillo CA, Shevach EM. Cutting edge: IL-2 is critically required for the in vitro activation of CD4+CD25+ T cell suppressor function. J Immunol 2004; 172:6519.

69 Fontenot JD, Rasmussen JP, Gavin MA, Rudensky AY. A function for interleukin 2 in Foxp-3-expressing regulatory T cells. Nat Immunol 2005; 6:1142. 